\title{
Hybrid 3-D Spectrophotometry of Emission-Line Nebulae at Ohio State
}

\author{
Richard W. Pogge \& Nancy Joanne Lame \\ Department of Astronomy, The Ohio State University, $174 \mathrm{~W} .18 \mathrm{th}$ \\ Ave, Columbus, $O H$ \{3210-1106, USA
}

\begin{abstract}
A program of 3-D spectrophotometry of emission nebulae being carried out at the Ohio State University will be described. We have had considerable success combining Fabry-Perot imaging, long-slit spectroscopy, and narrowband filter imaging into a hybrid 3-D spectroscopic approach that we have used to obtain detailed spectrophotometric maps of the density, temperature, extinction, and ionization in HII regions and Planetary Nebulae. The centerpiece instrument of this effort, the OSU Imaging Fabry-Perot Spectrograph (IFPS), will be described, and scientific results illustrative of our work will be presented.
\end{abstract}

\section{Introduction}

For the spectrophotometric study of spatially extended sources we are interested in deriving both spectral and spatial information. A particular science goal would be to measure the run of physical properties as a function of position in the object; e.g., mapping changes in density, temperature, ionization, or composition. In nebulae, subtle changes in structure can be better visualized if one can link those changes with spatial correlations over scales larger than a single detector pixel or aperture, making it easier to discern nebular ionization edges, filamentary structures embedded in the nebula, etc. Further information can be gained by obtaining full spectral information, and so being able to combine kinematic and nebular diagnostics to study gas flows, winds, or shocks. The requirements of a 3-D spectroscopic technique for nebulae comes down to a combination of good spectral and spatial coverage, and excellent relative spectrophotometry. The latter is especially critical, as most nebular diagnostics (e.g., for density or temperature) rely on rather subtle differences in emission-line ratios, and all diagnostics rely (to greater or lesser degree) on a precise estimate of the extinction in a nebula, which in large-scale nebulae can (and does) vary appreciably with position.

The ideal instrument for this work is the mythical 3-D spectrograph which gives us full spectral coverage across the observable band at every location in the telescope focal plane. Three traditional techniques for getting both spectral and spatial information have been applied, with various advantages and disadvantages: Long-8lit spectroscopy, interference filter imaging, and imaging FabryPerot interferometry (see Pogge 1992 for a detailed overview). Non-traditional techniques, like integral-field spectroscopy, are just now emerging, and have some problems of small fields of view (many papers this proceedings). None of these 
methods by themselves fulfill all of our requirements for true 3-D spectroscopy, but as I will argue in this contribution, a hybrid of these can approach the ideal. We have pursued this for the last 3 years at Ohio State with some success.

\section{Hybrid 3-D Spectrophotometry}

At the heart of the hybrid technique is the recognition that no single technique will do everything needed to address the problems of imaging spectroscopy. Long-slit spectra give excellent spectral coverage, but spatial coverage is discontinuous, entailing "painting" the object with the slit (intercalibration is hard but not impossible, see the SpectroNebulaGraph in this proceedings). Direct imaging through narrow filters gives full spatial coverage but only line fluxes, and that with a penalty of having to estimate, rather than measure, the underlying continuum using adjacent bandpasses. Fabry-Perots are capable of delivering a true spectrum at each field location continuously, but at the price of small free-spectral range and having to time-scan the wavelength axis, increasing sensitivity to changing observing conditions.

The essence of hybrid 3-D spectroscopy is to combine these approaches so as to exploit their strengths and mitigate their weaknesses. For example, in a study of Planetary Nebulae at OSU, we're using the following mix of techniques:

- Interference filter images for isolated line and continuum bands (e.g., $\mathrm{H} \beta$, $[O \mathrm{III}] \lambda 5007 \AA,[\mathrm{NII}] \lambda 5755 \AA, \mathrm{HeII} \lambda 4686 \AA$, and [O I] $\lambda 6300 \AA)$

- Imaging Fabry-Perot for blended lines (e.g., $\mathrm{H} \alpha+[\mathrm{N} \mathrm{II}]$ or $[\mathrm{S} \mathrm{II}] \lambda 6716+31$ ).

- Long-slit spectra for intercalibration of the imaging data and detailed followup of regions of interest to target faint lines.

Clearly how one chooses the mix depends on the scientific goals of the project. Since doing science is our ultimate goal and techniques are merely means to that end, there is much to be gained by adopting a less provincial attitude towards how one takes one's data.

\section{The OSU Imaging Fabry-Perot Spectrograph}

The centerpiece instrument is our Imaging Fabry-Perot Spectrograph (IFPS) built for use on the 1.8m Perkins telescope on Anderson Mesa near Flagstaff, Arizona (though its design allows it to travel to other telescopes easily). The IFPS provides a $6.5^{\prime} \mathrm{FOV}$ at $0.5 /$ pix onto a $15 \mu \mathrm{m}$ pixel CCD. Custom fused silica and $\mathrm{CaF}_{2}$ optics with broad-band thin-film AR coatings provides wavelength coverage of $\lambda \lambda 320-1000 \mathrm{~nm}$ with no degradation of efficiency or image quality. The six-position filter wheel can precision tilt interference filters for fine-tuning the bandpasses, and has a full set of nebular, redshifted, and broad-band filters. Etalons are located in collimated light at the pupil plane, and may be tilted to send all ghost reflections off into light-trapping baffles. Four Queensgate etalons are available: a low-resolution $\left(R_{\lambda}=1200\right)$, low-finesse $\left(N_{E}=35\right)$ etalon operating from $450-700 \mathrm{~nm}$, and three medium-resolution $\left(R_{\lambda}=4700\right)$, high-finesse $\left(N_{E}>60\right)$ etalons operating in the ranges $340-400,410-530$, and 


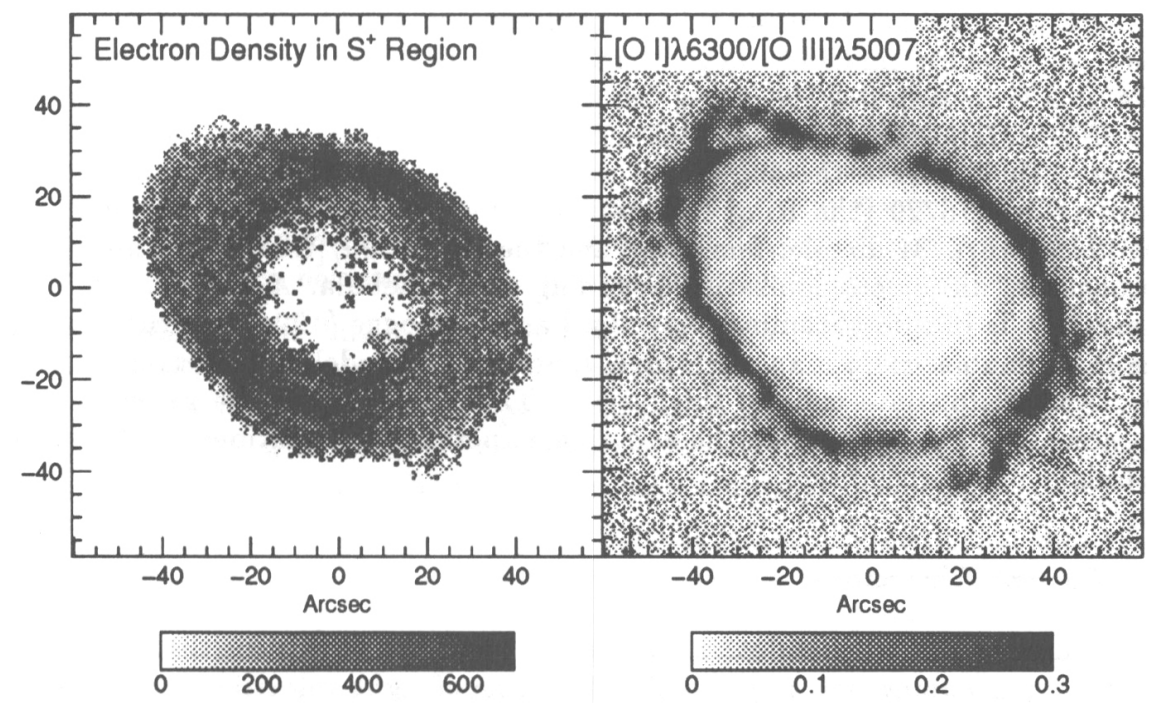

Figure 1. Density and excitation maps of the Ring Nebula obtained using the IFPS. The $[\mathrm{OI}] /[\mathrm{OIII}]$ excitation map uses filter images, while the [S II] density map is derived from Fabry-Perot spectrophotometry.

600-700nm. We have been using the IFPS in both its spectroscopic and direct imaging (etalon-less) modes for the last 4 years. A detailed description of the instrument is forthcoming in Pogge et al. (1994).

\section{Applications}

Our first efforts at hybrid 3-D spectroscopy used a low-resolution $\left(R_{\lambda}=1200\right)$ etalon in combination with long-slit spectra to obtain full-field imaging spectrophotometry of the core of the Orion Nebula (Pogge, Owen, \& Atwood 1992). We emphasize here that our F-P spectroscopy is true spectroscopy; we are not just imaging using the F-P as a tunable filter. We are relying on the spectroscopic information to allow accurate deblending of lines like [S II] $\lambda 6716+31$, a sensitive density diagnostic in nebulae that requires the ability to measure small difference in the doublet-line ratio. The scientific results of the study were to obtain maps of density, extinction, and excitation variations across the inner $6^{\prime}$ of the nebula in full spatial detail. A side benefit of this study were to demonstrate that we are able to achieve spectrophotometry with an F-P as good as that obtained with conventional long-slit techniques. With care in calibration and design of the instrument, and use of long-slit spectra to help with the intercalibration of the different line data sets, precision spectrophotometry is completely possible. 
A more complete application of our hybrid techniques has been our imaging spectrophotometric study of the structure of bright Planetary Nebulae. Here we combine imaging, long-slit spectra, and low-resolution F-P spectra. The goal it to map density (using the [S II] doublet ratio), temperature (using the [N II] $\lambda 5755,6548,6583$ lines), extinction, and excitation across four planetaries. To illustrate the success of our techniques $I$ show in Figure 1 two maps of NGC6720 (the Ring Nebula): the electron density computed using the $[\mathrm{S} \mathrm{II}] 6716 / 6731$ doublet-line ratio, and the ([O I $] 6300 /[\mathrm{OIII}] 5007)$ line ratio emphasizing the strong ionization bounding of this nebula. A full discussion of these data is in a forthcoming paper by Lame \& Pogge (1994). In the case of the [SII] doublet-ratio map, each pixel represents a model fit to a spectrum in the Fabry-Perot data cube of this object. The density structure revealed was unknown from previous long-slit and single-aperture observations of the Ring Nebula.

\section{Future Directions}

With our new medium-resolution etalons, we can begin to explore the kinematic dimension, mapping not only line flux, but line-of-sight velocities and velocity dispersion across nebulae. Our present projects include studies of shocked clouds in SNRs and HH objects. Future work, hopefully with access to larger telescopes, will focus on kinematically resolved imaging spectrophotometry of extended circumnuclear emission-line regions in Seyfert galaxies and nuclear starburst galaxies. As we have gained experience with our instrument and these techniques, we have so far achieved our principal goal: true imaging spectrophotometry.

Acknowledgments. The IFPS instrument was built by the OSU Astronomical Instrumentation Facility, particularly by the efforts of B. Atwood, P. Byard, \& T. O'Brien. IFPS instrument development was supported by NSF Grants AST-8822009 and AST-9112879. RWP thanks the colloquium organizers for a wonderfully productive meeting and their gracious hospitality.

\section{Discussion}

A. Chrysostomou: Did you make any assumptions in your calculations of e.g., your extinction map [for Orion], concerning the scattering of light by the walls of the molecular cloud?

$R$. Pogge: Yes, we did do a later analysis using scattering estimates based on those of Dufour et al. It changed the numbers to be sure, but it didn't change the overall appearance of the extinction map.

A. Chrysostomou: A comment, we have spectropolarimetric data for $\mathrm{H} \alpha, \mathrm{H} \beta$, and $[\mathrm{OIII}]$ which shows that over a large region of the [Orion] Nebula the line emission is scattered.

R. Pogge: You can also see scattering in ratios of the pure continuum maps we extract from our spectral data cubes. For example, many of the stars look like they have little halos around them. 
L. Deharveng: How do you perform the absolute calibration of your frames?

$R$. Pogge: We follow essentially the same procedures using standard stars and white-light flats described by Joss Bland-Hawthorn [in this volume]. For Orion, we also had a consistency check in the long-slit spectrophotometry published by Baldwin et al. [1991, ApJ, 374, 580]. Overall we can achieve an absolute precision of $\sim \pm 15 \%$, but relative photometry, which counts for the line ratios, is much better, as good as $\sim \pm 2 \%$ within a given data cube, and $\sim 5 \%$ at best for intercalibration between data cubes or images in different bands.

H. Castañeda: The line-ratio maps that you have shown are very useful to determine the physical properties of the nebula. Do you think it would be important to obtain line-ratio maps but based on a velocity criteria of selection (e.g., [O III]/H $\beta$ with both $2 \mathrm{D}$ maps at zero redshift)? Does OSU have some plans for this approach in the future (i.e., new etalons)?

$R$. Pogge: Yes, it is very important, and we're at least evolving in that direction. The work presented here was using our first, low-resolution $(R=1200)$ etalon, so we got no velocity information. We're starting new projects in which our collaborators are going to be bringing $R=10,000-20,000$ etalons to use in the IFPS, so we are starting to explore the kinematic axis.

\section{References}

Lame, N.J., \& Pogge, R.W. 1994, submitted to AJ.

Pogge, R.W. 1992, in Astronomical CCD Observing and Reduction Techniques, ed. S. Howell, ASP Conf. Ser., 23, 195

Pogge, R.W., Owen, J.M., \& Atwood, B. 1992, ApJ, 399, 147

Pogge, R.W., Atwood, B., Byard, P., O'Brien, T., \& Peterson, B. 1994, in preparation. 